Research paper

\title{
Analysis of TCR activation kinetics in primary human T cells upon focal or soluble stimulation
}

\author{
Boerge Arndt ${ }^{\mathrm{a}, 1,2}$, Mateusz Poltorak ${ }^{\mathrm{a}, 2}$, Bhavani S. Kowtharapu ${ }^{\mathrm{a}, 2,3}$, Peter Reichardt ${ }^{\mathrm{a}}$, \\ Lars Philipsen $^{\text {a }}$, Jonathan A. Lindquist ${ }^{\text {a }}$, Burkhart Schraven ${ }^{\text {a, b }}$, Luca Simeoni ${ }^{\text {a,* }}$ \\ a Otto-von-Guericke University, Institute of Molecular and Clinical Immunology, Leipziger Str. 44, 39120 Magdeburg, Germany \\ b Department of Immune Control, Helmholtz Centre for Infection Research, Inhoffenstrasse 7, Braunschweig 38124, Germany
}

\section{A R T I C L E I N F O}

Article history:

Received 24 September 2012

Received in revised form 14 November 2012

Accepted 14 November 2012

Available online 22 November 2012

\section{Keywords:}

T-cell activation

Signaling signature

Antibody stimulation

Physiological stimuli

Erk dynamics

TCR-mediated signaling

\begin{abstract}
A B S T R A C T
Signaling through the TCR is crucial for the generation of different cellular responses including proliferation, differentiation, and apoptosis. A growing body of evidence indicates that differences in the magnitude and the duration of the signal are critical determinants in eliciting cellular responses. Here, we have analyzed signaling dynamics induced upon TCR ligation in primary human $\mathrm{T}$ cells. We used CD3 antibodies either cross-linked in solution (sAbs) or immobilized on microbeads (iAbs), two widely employed methods to stimulate T cells in vitro. We show that classical sAbs stimulation induces a transient and abortive response, whereas iAbs induce sustained TCR-mediated signaling, resulting in productive T-cell responses previously observed only in antigen-specific murine systems. In summary, our analysis documents TCR signaling kinetics and suggests that iAbs are better suited for studying TCR-mediated signaling as they mimic antigen specific systems.
\end{abstract}

(c) 2012 Elsevier B.V. All rights reserved.

\section{Introduction}

Triggering of the $\mathrm{T}$ cell receptor (TCR) by pathogens activates an intricate network of signaling cascades that are both temporally and spatially regulated. These signaling events will lead to transcriptional activation, proliferation, and differentiation of $\mathrm{T}$ cells that will ultimately culminate in an immune response. Defects in signal transduction may lead to either T-cell hyperresponsiveness or impaired T-cell activation, which results in autoimmunity or immunodeficiency, respectively. Thus, the study of how TCR signaling is initiated, propagated, and translated into a cellular response is important for the understanding not only of physiological processes, but also of the molecular mechanisms underlying human diseases.

\footnotetext{
* Corresponding author. Tel.: + 4939167 17894; fax: + 493916715852 E-mail address: luca.simeoni@med.ovgu.de (L. Simeoni).

1 Present address: Diabetes Centre, Department of Medicine Innenstadt, Ludwig-Maximilian University, 80336 Munich, Germany.

2 B.A., M.P., and B.S.K. contributed equally to this work.

3 Present address: Vectorology and Experimental Gene Therapy, University of Rostock, 18057 Rostock, Germany.
}

To reproduce in vitro the signaling events occurring during physiological T-cell activation, immunologists need to pay particular attention in the choice of an appropriate stimulus. In vivo, T cells are activated upon contact with APCs expressing the agonistic peptide in the context of MHCmolecules. Therefore, the ideal method to activate $\mathrm{T}$ cells in vitro e.g. for biochemical analyses should mimic these conditions. Antigen-based systems, however, can only be used for the in vitro stimulation of monoclonal T-cell populations expressing a specific TCR, usually expressed on transgenic T cells, and are thus limited to mouse models.

Methods such as artificial antigen-presenting systems employing both cell-based and acellular technologies have been developed and are now being used to different extents in immunotherapy (Kim et al., 2004). However, to assess biochemical events during T-cell activation, soluble polyclonal stimuli such as agonistic antibodies against the TCR/CD3 complex, the co-receptors and/or the co-stimulatory molecules (i.e. CD4, CD28) are still largely used. For more than 30 years, this method has been extensively employed and indeed, has provided significant insight into the molecular 
events occurring during T-cell activation. However, stimulations with soluble ligands have clear limitations, as they fail to reproduce the extra dimension of a cell-cell contact and, more importantly, they do not induce T-cell responses (Berg et al., 1998; Wang et al., 2008). Thus, it is questionable whether TCR signaling induced upon stimulation with soluble Abs mirrors the physiologic signaling cascade leading to productive T-cell responses. Moreover, it appears somehow surprising that, despite the availability of tools that trigger T-cell activation, mimic APCs, and induce proliferation, the analyses of intracellular signaling pathways are still mainly performed by applying soluble ligands.

Alternative method of T-cell stimulation is based on microbeads coated with the same antibodies against TCR complex is an efficient tool that can be used to expand T cells ex vivo especially for immunotherapeutic applications (Levine et al., 1997; Garlie et al., 1999; Trickett and Kwan, 2003). It is generally believed that sAbs and iAbs have different functional properties. However, a comprehensive functional and biochemical characterization of the two widely employed methods to stimulate human T cells in vitro has not yet been performed. Additionally, it has also not yet been analyzed which of the two stimuli more closely mimics physiological stimulation and hence is more suitable for signaling studies. Therefore, we performed a systematic analysis of the signaling signatures induced by Abs against the TCR complex either cross-linked in solution (sAbs) or immobilized on microbeads (iAbs). Our analysis documents signaling kinetics and functional responses of iAbs and shows that they are better suited than sAbs for studying TCR-mediated signaling.

\section{Materials and methods}

\subsection{Human ethics}

Approval for these studies involving the analysis of TCR-mediated signaling in human T cells was obtained from the Ethics Committee of the Medical Faculty at the Otto-vonGuericke University, Magdeburg, Germany with the permission number [107/09]. Informed consent was obtained in writing in accordance with the Declaration of Helsinki.

\subsection{Cell purification}

Peripheral blood mononuclear cells were isolated by Ficoll gradient (Biochrom) centrifugation of heparinized blood collected from healthy volunteers. Total population of human T cells or $\mathrm{CD}^{+}$naïve and memory subpopulations were further purified by non-T cell depletion using specific $\mathrm{T}$ cell isolation kits (Miltenyi Biotec). The purity of T cells, determined by flow cytometry, was usually more than $96 \%$.

OT-II TCR transgenic or wild type mouse T cells were isolated using a Pan T-cell Isolation Kit and AutoMacs magnetic separation system (Miltenyi Biotec). APCs were prepared as follows: T and NK cells were stained using FITC-labeled CD3, CD4, CD8, and NK1.1 mAbs (BD Biosciences). After staining, T and NK cells were depleted using anti-FITC microbeads on an AutoMacs magnetic separation system. The remaining splenic cell suspension was used as APCs.

\subsection{T-cell stimulation}

After isolation, human T cells were cultured overnight in RPMI 1640 medium containing 10\% FCS (PAN Biotech) and $2 \mu \mathrm{g} / \mathrm{ml}$ Ciprobay (Bayer Schering Pharma). Successively, T cells were stimulated with either soluble or immobilized mAbs as follows. For soluble Ab stimulation, $2 \times 10^{6}$ cells were loaded with $10 \mu \mathrm{g} / \mathrm{ml}$ biotinylated anti-human CD3 (clone UCHT1, eBioscience) alone or in combination with $10 \mu \mathrm{g} / \mathrm{ml}$ biotinylated anti-human CD28 (clone CD28.2, eBioscience), and $10 \mu \mathrm{g} / \mathrm{ml}$ biotinylated anti-human CD4 (clone OKT4, eBioscience) mAbs as indicated in $100 \mu \mathrm{l}$ RPMI 1640 and for 15 min on ice. After washing, receptors were cross-linked by adding $25 \mu \mathrm{g} / \mathrm{ml}$ NeutrAvidin ${ }^{\mathrm{TM}}$ (Pierce). For microbead stimulation, SuperAvidin ${ }^{\mathrm{TM}}$-coated polystyrene microspheres $(\varnothing \sim 10 \mu \mathrm{m}$, Bangs Laboratories) were coated with biotinylated CD3 alone or in combination with CD28 and CD4 mAbs as indicated (10 $\mu \mathrm{g} / \mathrm{ml}$ each) for $30 \mathrm{~min}$ at $37{ }^{\circ} \mathrm{C}$ in PBS. Antibody-coated microbeads were washed twice with PBS, resuspended in RPMI 1640 and incubated with $T$ cells in a 1:1 ratio. Stimulation of $T$ cells was facilitated and synchronized by centrifugating samples for about $10 \mathrm{~s}$ at $1000 \mathrm{rpm}$. Biotinylated $\operatorname{IgG}_{2 \mathrm{a}}$ and $\operatorname{IgG}_{1}$ mouse immunoglobulins (eBioscience) were used as a control. Stimulation with plate-bound antibodies was performed as follows. $10 \mu \mathrm{g} / \mathrm{ml}$ of CD3 and $10 \mu \mathrm{g} / \mathrm{ml} \mathrm{CD28} \mathrm{Abs} \mathrm{were} \mathrm{coated} \mathrm{on} \mathrm{U-bottomed}$ 96-well plates for $2 \mathrm{~h}$ at $37^{\circ} \mathrm{C}$. After washing, $1 \times 10^{6}$ cells were added to the plates, rapidly centrifuged at $1000 \mathrm{rpm}$ and incubated at $37{ }^{\circ} \mathrm{C}$ for the indicated time points.

Mouse $\mathrm{T}$ cells were stimulated with either soluble or immobilized $\beta T C R \times C D 28 \mathrm{mAbs}$ and processed as described above. For stimulations, $10 \mu \mathrm{g} / \mathrm{ml}$ of soluble or immobilized BTCR (clone H57-597, BD Biosciences) and CD28 (clone 37.51, BD Biosciences) mAbs were used. OT-II TCR transgenic T cells $\left(1 \times 10^{6}\right)$ were stimulated with $\left(2 \times 10^{6}\right)$ APCs that were either left unloaded or loaded with $5 \mu \mathrm{g} / \mathrm{ml}$ of $\mathrm{OVA}_{323-339}$ for the indicated period of time.

\subsection{Western blotting}

T cells were lysed in buffer containing 1\% lauryl maltoside (N-dodecyl $\beta$-maltoside), 1\% NP-40, 1 mM Na $\mathrm{VO}_{4}, 1 \mathrm{mM}$ PMSF, $10 \mathrm{mM} \mathrm{NaF}, 10 \mathrm{mM}$ EDTA, $50 \mathrm{mM}$ Tris pH 7.5, and $150 \mathrm{mM} \mathrm{NaCl}$. Post-nuclear lysates were separated by SDS-PAGE and transferred onto nitrocellulose membranes. Membranes were probed with the indicated primary antibodies and the appropriate HRP-conjugated secondary antibodies (Dianova) and developed using the ECL detection system (Amersham Pharmacia). The following antibodies were used for Western blotting in this study: anti-phospho(p)- $\mathrm{T}^{202} / \mathrm{Y}^{204}$ Erk1/2, anti-pY ${ }^{171}$ LAT, anti-pY ${ }^{783}$ PLC- $\gamma 1$, anti-pS ${ }^{338}$-c-Raf, anti-pS ${ }^{217 / 221}$ MEK1/2 (all from Cell Signaling Technology), and anti- $\beta$-actin (clone AC-15) (Sigma-Aldrich). For quantifications of the Western blots, the intensity of the detected bands was acquired using the Kodak Image Station 2000R and analysis was performed using 1D ImageQuant software (Kodak).

\subsection{In vitro assays}

Proliferation experiments were carried out in 96-well plates (Costar). T cells were labeled with $2.5 \mu \mathrm{M}$ CFSE (Molecular 
Probes) for $10 \mathrm{~min}$ at $37^{\circ} \mathrm{C}$. After washing, $2 \times 10^{5}$ cells were seeded in a total volume of $200 \mu \mathrm{l}$ to each well and cultured in RPMI (supplemented with 10\% FCS and antibiotics). Human $\mathrm{T}$ cells were either left unstimulated or were stimulated with soluble or immobilized $\mathrm{CD} 3$ alone, $\mathrm{CD} 3 \times \mathrm{CD} 28$, and $\mathrm{CD} 3 \times \mathrm{CD} 28 \times \mathrm{CD} 4 \mathrm{mAbs}$ as indicated above (see T-cell stimulation). Mouse $\mathrm{T}$ cells were either left unstimulated or stimulated with $10 \mu \mathrm{g} / \mathrm{ml}$ of soluble or immobilized BTCR (clone H57-597, BD Biosciences) and CD28 (clone 37.51, BD Biosciences) mAbs. T cells were cultured for $72 \mathrm{~h}$ at $37{ }^{\circ} \mathrm{C}$, $5 \% \mathrm{CO}_{2}$. Proliferation was assessed by CFSE dilution using a FACS-Calibur and the CellQuest software (BD Biosciences) or BD LSRFortessa, FACSDiva Software 6.1.3 (BD Biosciences), and FlowJo 7.6.5 (Tree Star, Inc.).

To measure proliferation of lymph node cells isolated from OT-II transgenic mice $1 \times 10^{5}$ cells/well were cultured for 2 days in RPMI 1640 medium (supplemented with 10\% FCS, antibiotics, 2-ME) in U-bottom 96-well plates (Costar) in the presence of $2 \times 10^{5} \mathrm{~T}$ cell-depleted irradiated splenocytes from wild-type mice loaded with increasing amount of $\mathrm{OVA}_{323-339}$ peptide. Cells were pulsed with $1 \mu \mathrm{Ci}\left[{ }^{3} \mathrm{H}\right]$-thymidine per well during the last $8 \mathrm{~h}$ and harvested using a PHD cell harvester (Inotech AG, Basel, Switzerland). Thymidine incorporation was measured by liquid scintillation counting.

To determine $\mathrm{T}$-cell activation, human $\mathrm{T}$ cells were stimulated as described above. After $24 \mathrm{~h}$, T cells were stained with either FITC- or PE-labeled mAbs against CD25 and CD69 (BD Biosciences), respectively. Cell associated fluorescence was analyzed by flow cytometry.

To quantify cell survival under different stimulation conditions, human T cells were stimulated with either soluble or immobilized mAbs as described above, and resuspended in RPMI supplemented with $10 \%$ FCS at a density of $1 \times 10^{6}$ cells $/ \mathrm{ml}$ in a 24-well tissue culture plate. Cells were harvested after $24 \mathrm{~h}$ and the percentage of cells undergoing apoptosis were measured by flow cytometry using FITC-annexin V and propidium iodide (PI) (rh annexin V/FITC kit; Bender MedSystems) according to manufacturer's instructions.

To measure the antibody binding capacity, $1 \times 10^{6}$ microbeads were coated with either CD3 alone or in combination with CD28 and CD4 mAbs as described above. Subsequently, microbeads were washed and stained with FITC-conjugated anti-mouse antibody (Jackson ImmunoResearch Laboratories). Beads associated fluorescence was analyzed by flow cytometry.

\section{6. $T$ cell/microbead binding}

Primary human T cells were mixed with the CD3 $\times$ CD28 coated microbeads in a $1: 1$ ratio, rapidly centrifuged at $1000 \mathrm{rpm}$ and incubated at $37{ }^{\circ} \mathrm{C}$ for the indicated time points in a $3 \mathrm{ml}$ round-bottom tube. Subsequently, the samples were gently resuspended in $500 \mu \mathrm{PBS}$ and analyzed on a FACS Calibur. Microbeads coated with $\operatorname{IgG}_{2 a}$ and $\operatorname{IgG}_{1}$ were used as a control. T cell/microbead conjugates were also counted by using a hemocytometer. Single-cell imaging was done on a Leica microscope equipped with a $20 \times$ imaging system from Visitron Systems Imaging $\mathrm{GmbH}$.

\subsection{Florescence microscopy}

Cell stimulation was performed as indicated above. Subsequently, cells were transferred to PLL-coated cover slides and allowed to settle down for $10 \mathrm{~min}$ at $4{ }^{\circ} \mathrm{C}$ before fixation with $2 \%$ para-formaldehyde. The samples were permeabilized with $0.2 \%$ TritonX100 for 10 min and blocked with $1 \%$ BSA for 30 min. Cell conjugates were stained with propidium iodide (Sigma Aldrich) and anti-pY ${ }^{142} \mathrm{CD} 3 \zeta$-Alexa488 antibody (BD Pharmigen), and placed on the stage of an inverted wide-field fluorescence microscope (Leica DM IRE2; 63× oil lens NA 1.40). The fluorescent signals and the corresponding phase contrast image were acquired by a cooled charge-coupled device camera (Apogee KX4; Apogee Instruments, Roseville, CA, $2 \times$ binning). Fluorescence images were corrected for illumination faults using flat-field correction.

\section{Results}

3.1. iAbs induce activation and proliferation, whereas, sAbs result in $T$-cell unresponsiveness

It is generally accepted that soluble antibodies cannot induce productive T-cell responses. Indeed, we and others a

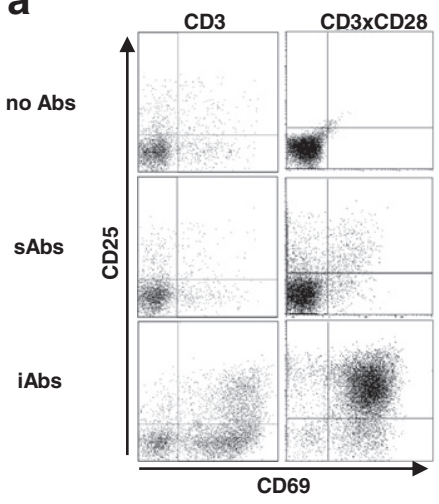

b

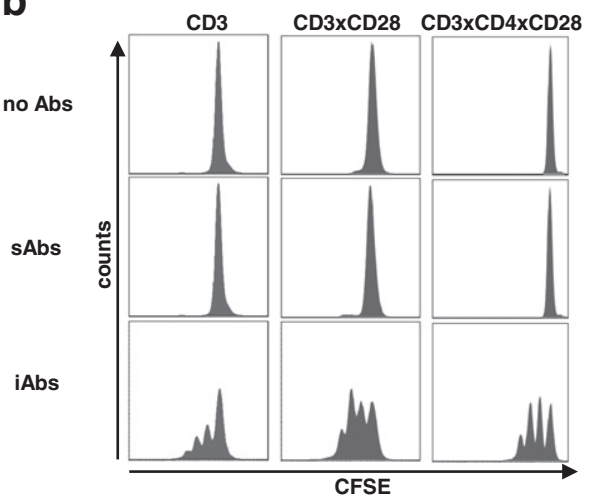

C

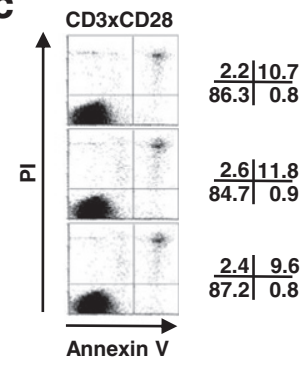

Fig. 1. Analysis of the functional effects of sAbs and iAbs. Purified human T cells were treated as indicated. (a) 24 h after stimulation, the activation of T cells was analyzed by staining with CD25 and CD69 and flow cytometry. (b) T cells were labeled with CFSE and stimulated as indicated. Proliferation was assessed after 72 h by analyzing CFSE content on a FACS Calibur. (c) T cells were stimulated as indicated for 24 h. Cell survival was assessed by FITC-annexin V and PI staining. One representative experiment of at least three independent experiments is shown. Numbers in c indicate the proportion of cells in each quadrant. 
have demonstrated that OT-I transgenic T cells and cytotoxic T-lymphocyte clones are not activated and do not differentiate upon stimulation with antibodies cross-linked in suspension (Berg et al., 1998; Wang et al., 2008). Here, we have stimulated primary human $\mathrm{T}$ cells with either sAbs or iAbs and analyzed their functional responses. Fig. 1 shows that treatment of T cells with iAbs led to an expression of CD25 and CD69 and to a proliferative response. Conversely, treatment with sAbs (CD3 alone or in combination with CD28) failed to induce T-cell activation as indicated by the absence of CD69 and CD25 expression (Fig. 1a). In agreement with these data, proliferation assays revealed that $T$ cells stimulated with sAbs (CD3 alone or in combination with CD28 and CD4) also did not proliferate (Fig. 1b).

To exclude the possibility that the lack of proliferation depends on the particular clone of antibody used for stimulation, we repeated the experiments depicted in Fig. 1 using two other CD3 mAbs of different Ig classes (OKT3 and MEM92). Moreover, we also tested different concentrations of soluble anti-CD3 (UCHT-1) (ranging from $0.1 \mu \mathrm{g} / \mathrm{ml}$ to $10 \mu \mathrm{g} / \mathrm{ml}$ ) together with various concentrations of anti-CD28 (ranging from $0.1 \mu \mathrm{g} / \mathrm{ml}$ to $10 \mu \mathrm{g} / \mathrm{ml}$ ) in different ratios in the presence or absence of anti-CD4. Under all conditions tested, human $\mathrm{T}$ cells did not proliferate in response to stimulation with cross-linked soluble Abs (Fig. 1b, Supplementary Fig. 1, and data not shown).

Finally, similar to human T cells, also the stimulation of mouse $\mathrm{T}$ cells with $\mathrm{TCR} \beta \times \mathrm{CD} 28 \mathrm{mAbs}$ immobilized on microbeads resulted in proliferation, whereas, stimulation with the same antibodies cross-linked in suspension did not induce productive T-cell response (Supplementary Fig. 2). Similar stimulation conditions also failed to induce proliferation in OT-I transgenic T cells (Wang et al., 2008).

We have previously shown that cross-linked soluble CD3 $\times$ CD8 antibodies induce apoptosis rather than proliferation in OT-I TCR transgenic CD8 ${ }^{+}$mouse T cells (Wang et al., 2008). To analyze whether stimulation of human T cells with sAbs may also induce apoptosis, peripheral $\mathrm{T}$ cells were stimulated overnight and assayed for annexin $\mathrm{V}$ expression. Fig. 1c shows that, in contrast to mouse, stimulation of human T cells with sAbs did not induce apoptosis. Thus, despite the fact that sAbs are commonly used to activate proximal and distal TCR-mediated signaling pathways, they are unable to induce productive T-cell activation and proliferation.

\section{2. iAbs efficiently stimulate T cells}

To date, little is known about the signaling properties of mAbs coated to microbeads. We initially established a protocol to efficiently stimulate $\mathrm{T}$ cells with iAbs. For stimulation, we employed $10 \mu \mathrm{m}$ microbeads that were coated with SuperAvidin. This gives the possibility to bind any biotin-labeled antibody or recombinant protein on the surface of the microbeads. For this investigation, we used biotinylated $\mathrm{CD} 3 \varepsilon$ and $\mathrm{CD} 28 \mathrm{mAbs}$ unless indicated otherwise. Microbeads were coated with $10 \mu \mathrm{g} / \mathrm{ml}$ biotinylated CD3 and CD28 mAbs. Microbeads loaded with non-relevant antibodies of the same isotype were used as control. To induce rapid and synchronous cell contact with the beads, we performed a brief centrifugation ( $5 \mathrm{~s}$ at $1000 \mathrm{rpm}$ ) in a small volume $(100 \mu \mathrm{l})$. Aliquots of the samples were taken at regular time intervals and analyzed on a FACS Calibur. Fig. 2a shows that $\mathrm{T}$ cells and microbeads have clearly different physical parameters and can be easily visualized in FSC/SSC dot plots. The data also demonstrate that a significant number of cells associated with microbeads coated with $\mathrm{CD} 3 \times \mathrm{CD} 28$ Abs and appeared as a new population indicated in the gate $R_{T c}+m$ (Fig. 2b). Conversely, in the control samples the emergence of a new population in the $R_{T c}+m$ gate is not detected (Fig. 2b, upper panels), thus indicating that microbeads loaded with isotype controls do not bind to T cells. Successively, to quantify T cell-microbead association, we calculated the ratio $R_{a}$ of the number of $\mathrm{T}$ cell/microbead conjugates (events in the $\mathrm{R}_{\mathrm{Tc}}+\mathrm{m}$ gate) to the total number of $\mathrm{T}$ cells (events in the $\mathrm{R}_{\mathrm{Tc}}$ gate). We found that a high fraction of the cells was in contact with microbeads already after 5 min incubation, with $R_{a}$ approximately 0.2 (Fig. 2c). After $15 \mathrm{~min}$ incubation, the proportion of T cells in contact with microbeads increased up to $50 \%\left(R_{a}=\right.$ $0.5)$. Afterwards, the $R_{a}$ slightly decreased and stabilized at values close to 0.3. A similar association rate between Jurkat $\mathrm{T}$ cells and CD3-coated micrometric particles has been recently described (Carpentier et al., 2009). To further address this issue and to check whether mechanical stress during flow cytometry measurements resulted in the disruption of $\mathrm{T}$ cell/bead conjugates, we performed additional analyses. We initially assessed cell/bead conjugates using light microscopy (Fig. 2d). We found that a high number of $T$ cells rapidly established contact with iAbs. We next quantified cell/bead conjugates by manual counting in a hemocytometer. The data depicted in Fig. 2e show that around $50-70 \%$ of the cells conjugate with beads. As the proportion of $\mathrm{T}$ cell/microbead conjugates was slightly higher than this presented in Fig. 2c, this suggests that some contacts with beads were lost during flow cytometry. Collectively, these data demonstrate that a very high number of iAbs interact with T cells, thus ensuring a focal stimulation.

Additionally, we have analyzed formation of signaling microclusters in the T cell/bead contact zone by fluorescence microscopy. As shown in Supplementary Fig. 3, both physiological and iAbs stimulation induce the formation of signaling microclusters.

We next quantified the effectiveness of the stimulation by analyzing the proportion of cells displaying phosphorylated Erk1/2. The data presented in Fig. 2f confirmed that the cell/ bead contact indeed resulted in T-cell stimulation as the proportions of $\mathrm{T}$ cells in contact with beads and those displaying intracellular phospho-Erk1/2 were similar. Similar proportions of activated $\mathrm{T}$ cells were also obtained upon stimulation with sAbs (Fig. 2g). Thus, the efficiency of iAbs and sAbs in stimulating $\mathrm{T}$ cells appears to be comparable.

Next, we have measured fluorescence of beads loaded with either $\mathrm{CD} 3$ alone or $\mathrm{CD} 3 \times \mathrm{CD} 28$ and $\mathrm{CD} 3 \times \mathrm{CD} 4 \times \mathrm{CD} 28$ to analyze binding capacity of microbeads. We have observed a consistent increase in the fluorescence of beads loaded with single vs. beads loaded with multiple mAbs. These data indicate that the coating with CD3 and CD3 $\times$ CD28 mAbs are not sufficient to saturate the microbeads (Supplementary Fig. 4).

\subsection{Sustained versus transient activation kinetics in TCR-mediated signaling induced by $i A b s$ and $s A b s$, respectively}

As sAbs and iAbs induce markedly different cellular outcomes, we next performed a comparative analysis of 

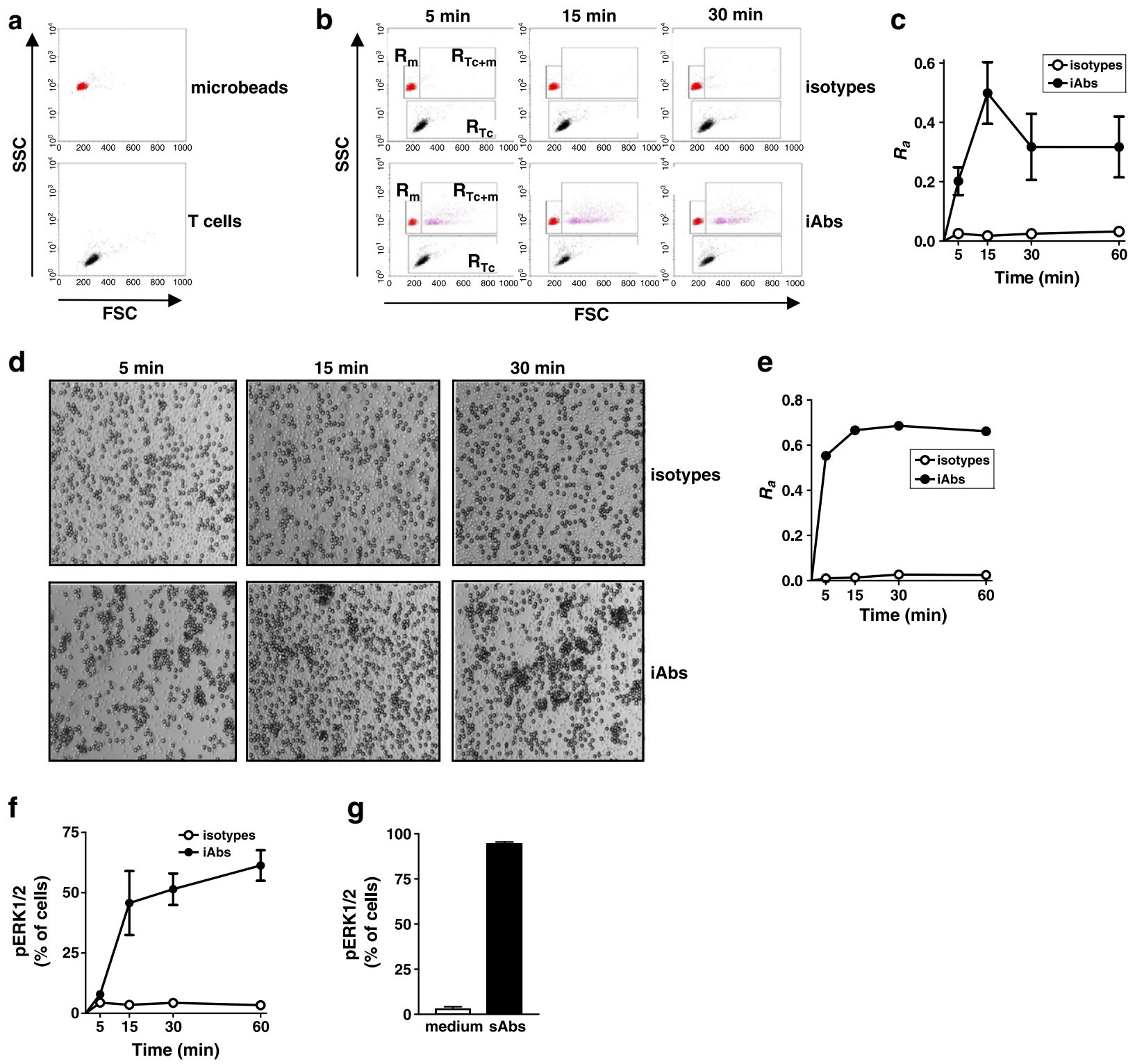

Fig. 2. Analyses of the $\mathrm{T}$ cell/microbead binding. (a) $\mathrm{T}$ cells and microbeads alone were analyzed by side scattering versus forward scattering dot plots. (b) Subsequently, T cells and microbeads loaded either with isotype controls (isotypes, upper panels) or CD3 $\times$ CD28 mAbs (iAbs, lower panels) were brought into contact for the indicated time periods. T cell/microbead conjugates (RTc $+\mathrm{m}$ ) are clearly distinct from free T cells (RTc) and microbeads (Rm). (c) Statistical analysis of the ratio $\left(R_{a}\right)$ between the numbers of T cell/microbead conjugates (events in the RTc $+\mathrm{m}$ gate) to the total number of unconjugated T cells (events in the RTc gate) is presented. (d) Transmission light images of T cells incubated with microbeads loaded either with isotype controls (isotypes, upper panels) or CD3xCD28 mAbs (iAbs, lower panels) are shown. (e) T cell/microbead conjugates were diluted and manually counted in a hemocytometer. The graph shows statistical analysis of the ratio $\left(R_{a}\right)$ between the numbers of T cell/microbead conjugates to the total number of T cells from three independent experiments. Human $T$ cells were purified and stimulated with beads loaded either with CD3xCD28 mAbs (iAbs) or isotype control (isotypes) for the indicated time points. (f) The level of pErk1/2 as marker of activation was measured by intracellular staining. Data represent the mean proportions of pErk1/2 positive cells \pm SEM of 3 independent experiments. (g) Human T cells were purified and either left unstimulated (medium) or were treated with CD3 $\times$ CD28 mAbs cross-linked in solution ( $\mathrm{sAbs}$ ) for $2 \mathrm{~min}$. The graph shows the mean proportions of pErk1/2 positive cells \pm SEM from 3 independent experiments.

their signaling signatures in primary human T cells. We found that the magnitude and the duration of the signal induced by iAbs were strikingly different to those induced by sAbs (Fig. 3). Indeed, stimulation with sAbs (CD3 alone or in combination with $\mathrm{CD} 28$ or CD4) resulted in strong and transient activation of molecules involved in TCR-mediated proximal signaling, such as LAT and PLC $\gamma 1$, whereas iAbs stimulation led to weaker but sustained phosphorylation
(Fig. 3a). As an example of downstream pathway activated via the TCR, we investigated the phosphorylation levels of molecules in the Ras-Erk signaling cascade. Despite the fact that the magnitude of the phosphorylation of c-Raf, MEK1/2, and Erk1/2 appeared to be comparable in both types of stimulation, their activation was sustained upon iAbs treatment (Fig. 3a). Similar activation kinetics were also observed when purified mouse splenic T cells were stimulated with 
a

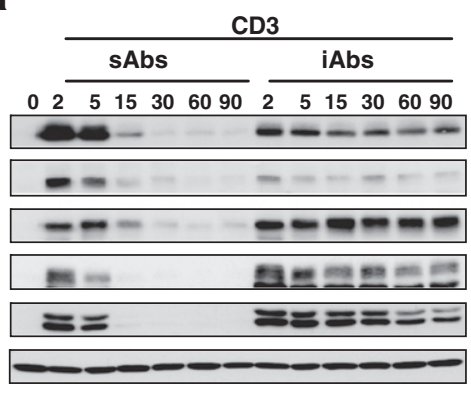

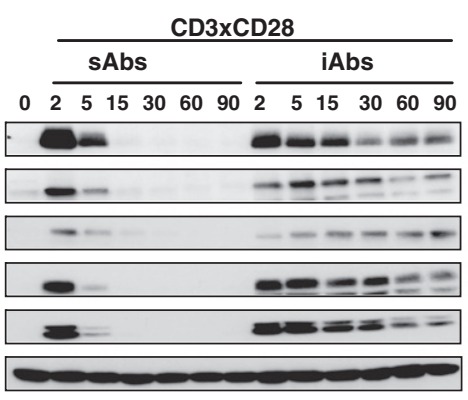

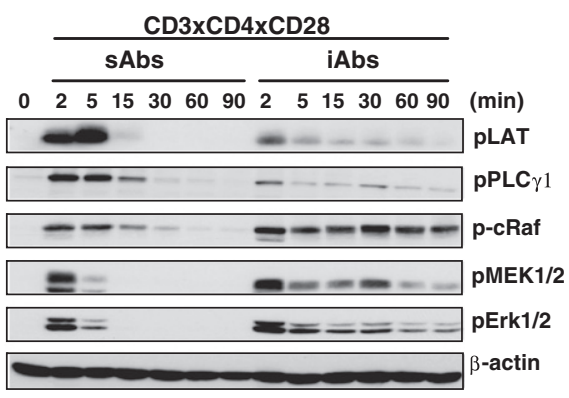

b

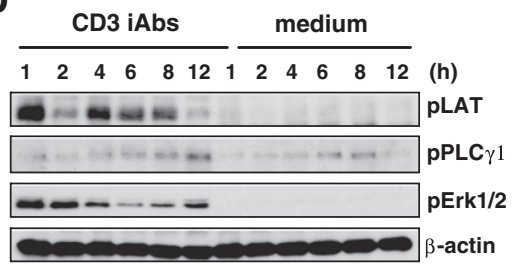

Fig. 3. sAbs induce transient, whereas, iAbs induce sustained signaling. (a,b) Purified human T cells were treated with either soluble (sAbs) or immobilized (iAbs) mAbs as indicated. Samples were analyzed by Western blotting using the indicated Abs. For each condition one representative immunoblot of at least 4 independent experiments is shown.

TCR $\beta \times$ CD28 Abs immobilized on microbeads or cross-linked in solution (Supplementary Fig. 5), suggesting that TCRmediated signaling induced by antibodies immobilized on beads is not dependent on the antibody clones used for stimulation.

Next, we compared activation kinetics of purified naïve and memory $\mathrm{CD} 4^{+} \mathrm{T}$ cells upon stimulation with sAbs and iAbs. Similar to unfractionated $\mathrm{T}$ cells, both $\mathrm{CD} 4^{+} \mathrm{T}$-cell subsets displayed a transient activation upon sAbs stimulation, but sustained signaling upon treatment with iAbs (Supplementary Fig. 6). Thus, it appears that the signaling signatures of sAbs and iAbs are independent of the differentiation status of the cells.

Finally, we analyzed the signaling signatures induced by plate-bound antibodies, which also induce T-cell activation and proliferation. As shown in Supplementary Fig. 7, we found that plate-bound Abs have signaling kinetics similar to iAbs.

In summary, we show that stimulation with sAbs vs. iAbs induces different activation kinetics of both TCR proximal and distal signaling molecules. We believe that the duration of the signal may be the determinant factor for the generation of cellular responses.

\subsection{Signaling dynamics induced by iAbs are similar to those induced by stimulation with APC}

We next analyzed the signaling kinetics induced by physiological stimuli. We used $\mathrm{CD}^{+} \mathrm{T}$ cells isolated from OT-II transgenic mice that were stimulated with APCs alone as control or with APCs loaded with the OVA $323-339$ peptide. Stimulation with OVA-loaded APC proved to be effective method to generate productive response of OT-II transgenic T cells in the dose dependent manner (Fig. 4a). Fig. 4b shows that stimulation with peptide loaded APCs, but not with control APCs, induces sustained phosphorylation of LAT, PLC $\gamma 1$, and Erk1/2 in OT-II transgenic T cells. These data further corroborate our previous observations showing that the stimulation of $\mathrm{CD}^{+}{ }^{+} \mathrm{T}$ cells from OT-I transgenic mice with physiological ligands also induced sustained phosphorylation of signaling molecules such as Erk1/2 and PLC $\gamma-1$ (Wang et al., 2008). It is important to note that, iAbs elicit effects that are comparable to those obtained with a physiological stimulation (Fig. 4 and (Wang et al., 2008)).

Collectively, these data show that stimulation of human $\mathrm{T}$ cells with immobilized Abs induces activation kinetics similar to those induced by physiological ligands in mouse TCR transgenic systems and that sustained activation of TCR signaling correlates with proliferation. In conclusion, it appears that iAbs mimic more closely physiological stimulation than sAbs and hence are better suited to study signaling correlating with productive T-cell responses.

\section{Discussion}

Current stimulation protocols to study T-cell signaling are mainly based on agonistic antibodies cross-linked in solution. This method has several advantages such as low costs, easy handling, high reproducibility, effective activation of signaling molecules, polyclonal activity and, most of all, it is well-characterized. However, despite its routine use to study signaling during T-cell activation, it is known that sAbs induce only a transient activation signal that results in an abortive response. Moreover, soluble antibodies do not reproduce the cell-cell contact induced by an APC and therefore fail to mimic at least one crucial event occurring during T-cell activation.

Here, we have analyzed whether Abs immobilized on microbeads, which mimic APCs and induce proliferation, represent an alternative and more suitable method to sAbs 
a

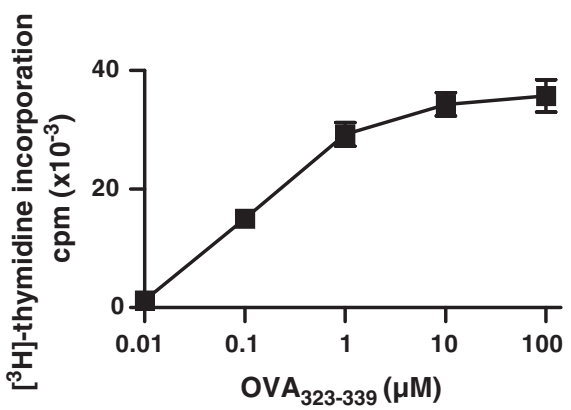

b

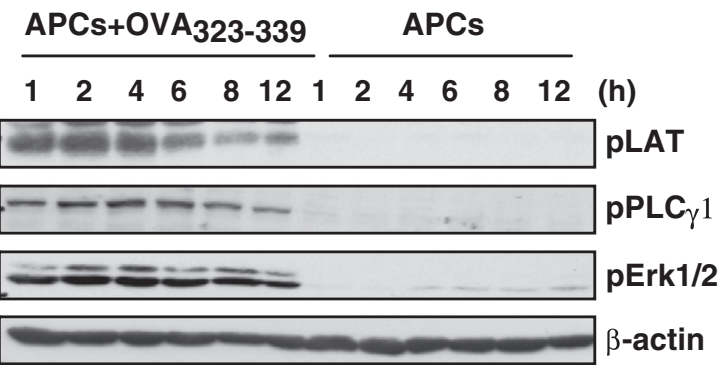

Fig. 4. OT-II transgenic $T$ cells stimulated with OVA peptide present sustained activation dynamics. (a) Lymph node cells isolated from OT-II transgenic mice were stimulated in the presence of irradiated APCs pulsed with increasing amount of $\mathrm{OVA}_{323-339}$ peptide. Proliferation was measured after $72 \mathrm{~h}$. Data derive from 3 mice. (b) Purified mouse splenic OT-II transgenic T cells were stimulated with APCs either left unloaded or loaded with the agonist peptide $\mathrm{OVA}_{323-339}$ for the indicated time points. Samples were analyzed by Western blotting using the Abs indicated.

for the stimulation of $\mathrm{T}$ cells for signaling studies. We further compared the kinetics of central signaling events induced by Abs immobilized on microbeads to those generated by Abs cross-linked in solution. We document profound signaling differences between these methods of stimulation.

One of the most important findings is that the sustained activation induced by iAbs recapitulated signaling events observed in antigen-specific systems. In fact, we show that T cells stimulated with APCs loaded with the OVA-peptide display similar activation kinetics as $T$ cells stimulated with beads. Moreover, we have previously shown that $\mathrm{H}-2 \mathrm{~K}^{\mathrm{b}}$ molecules loaded with the SIINFEKL peptide triggered prolonged activation of ZAP-70, LAT, PLC $\gamma$-1, and Erk1/2 and also induced proliferation in OT-I transgenic T cells (Wang et al., 2008).

In addition to the immobilization on beads, Abs can also be bound to cell culture plates. Similarly to iAbs, also platebound antibodies induce functional responses. Previous studies have shown that plate-bound, but not soluble CD3 Abs induce sustained TCR-mediated tyrosine phosphorylation and MAPK activation in murine CTL clones (Berg et al., 1998; Puente et al., 2000, 2006). However, the relevance of these studies is limited. They utilize $\mathrm{CD} 8^{+} \mathrm{T}$-cell clones, which were expanded in the presence of IL-2 and were stimulated with CD3 Abs alone in the absence of co-stimulation. Therefore, it is questionable whether these data also apply to primary human $\mathrm{T}$ cells. Conversely, in our studies we have provided an extensive biochemical characterization of signaling networks upon stimulation with either sAbs or iAbs in total human peripheral T cells as well as in naïve and memory CD4 ${ }^{+} \mathrm{T}$ cells. To our knowledge similar studies describing the signaling properties of Abs immobilized either on beads or cell culture plates have not been performed. Despite the fact that both iAbs and plate-bound antibodies induce functional responses and likely similar signaling kinetics, antibodies immobilized on microbeads allow an easier handling of the samples during stimulation compared to plate-bound antibodies. Therefore, we believe that iAbs are a valid method of stimulation that allows easy and large scale biochemical analyses of TCR-mediated signaling events.

The availability of a method that mimics antigen-specific systems is of particular importance to study primary human T cells, which cannot be stimulated on a large scale by antigenspecific systems. Data from primary human $\mathrm{T}$ cells are still scarce and most of our knowledge on signaling in human $T$ cells is based upon data obtained from the analysis of lymphoid cell lines that were stimulated with cross-linked soluble mAbs. Experiments employing primary human cells stimulated with more physiological systems are not only necessary to study the basis of T-cell function, but are also essential to understand the molecular mechanisms of human diseases such as autoimmunity and cancerogenesis.

In summary, our studies clearly show that iAbs are a valid alternative to sAbs for stimulation of $\mathrm{T}$ cells for signaling analysis. First, iAbs are easy and ready-to-use. Second, iAbs mimic signaling kinetics described in antigen-specific systems. Third, iAbs trigger signaling pathways correlating with T-cell activation and proliferation.

\section{Acknowledgments}

We are grateful to Tilo Bayer for critically reading the manuscript and helpful discussion and to Nicole Jüling, Ines Meinert, Stefanie Holze, and Guido Höbbel for their excellent technical assistance.

The work was supported by grants from the German Research Foundation (DFG), FOR-521 [SI861/1], GRK-1167 [TP12] and SFB-854 [TP19].

\section{Appendix A. Supplementary data}

Supplementary data to this article can be found online at http://dx.doi.org/10.1016/j.jim.2012.11.006.

\section{References}

Berg, N.N., Puente, L.G., Dawicki, W., Ostergaard, H.L., 1998. Sustained TCR signaling is required for mitogen-activated protein kinase activation and degranulation by cytotoxic T lymphocytes. J. Immunol. 161, 2919.

Carpentier, B., Pierobon, P., Hivroz, C., Henry, N., 2009. T-cell artificial focal triggering tools: linking surface interactions with cell response. PLoS One 4, e4784.

Garlie, N.K., LeFever, A.V., Siebenlist, R.E., Levine, B.L., June, C.H., Lum, L.G., 1999. T cells coactivated with immobilized anti-CD3 and anti-CD28 as potential immunotherapy for cancer. J. Immunother. 22, 336.

Kim, J.V., Latouche, J.B., Riviere, I., Sadelain, M., 2004. The ABCs of artificial antigen presentation. Nat. Biotechnol. 22, 403.

Levine, B.L., Bernstein, W.B., Connors, M., Craighead, N., Lindsten, T., Thompson, C.B., June, C.H., 1997. Effects of CD28 costimulation on long-term proliferation of $\mathrm{CD} 4+\mathrm{T}$ cells in the absence of exogenous feeder cells. J. Immunol. 159, 5921. 
Puente, L.G., Stone, J.C. Ostergaard, H.L., 2000. Evidence for protein kinase $\mathrm{C}$-dependent and -independent activation of mitogen-activated protein kinase in T cells: potential role of additional diacylglycerol binding proteins. J. Immunol. 165, 6865.

Puente, L.G., He, J.S., Ostergaard, H.L., 2006. A novel PKC regulates ERK activation and degranulation of cytotoxic $\mathrm{T}$ lymphocytes: plasticity in PKC regulation of ERK. Eur. J. Immunol. 36, 1009.
Trickett, A., Kwan, Y.L., 2003. T cell stimulation and expansion using antiCD3/CD28 beads. J. Immunol. Methods 275, 251.

Wang, X., Simeoni, L., Lindquist, J.A., Saez-Rodriguez, J., Ambach, A., Gilles, E.D., Kliche, S., Schraven, B., 2008. Dynamics of proximal signaling events after TCR/CD8-mediated induction of proliferation or apoptosis in mature CD8 + T cells. J. Immunol. 180, 6703. 